\title{
Student Design, Development and Operations of Small Satellites at the United States Air Force Academy
}

\author{
Kenneth E. Siegenthaler, Jerry J. Sellers, \\ David J. Richie, and Timothy J. Lawrence \\ Department of Astronautics \\ United States Air Force Academy
}

\begin{abstract}
The FalconSAT program is a unique, dynamic small-satellite research program that serves as a capstone course for Astronautical Engineering majors at the United States Air Force Academy. The goal of the program is to give students the opportunity to "learn space by doing space." The program results in a satellite launched into space every two to three years. It is conducted in the same manner required of any civilian company delivering a satellite for a NASA/Air Force launch. In addition to the design and construction of the satellites, students must meet all of the Department of Defense (DoD) milestones, including preparing and briefing the Alternative Systems Review (ASR), Preliminary Design Review (PDR), Critical Design Review (CDR), and Product Acceptance Demonstration (PAD). These reviews are given to and evaluated by members of the civilian aerospace community and scientists and engineers from U.S. Air Force space organizations outside of the Academy. Each student is required to become familiar with the functioning of the payload and all of the subsystems. The average student participates in design, clean-room construction, shake and bake-out testing, ground station operations, program management, and presents review briefings during the two-semester course. The students also prepare and brief the proposed experimental payload briefings to the DoD Space Experiments Review Board (SERB), competing on a level playing field with all of the other civilian and military proposals. This paper discusses the current status of the FalconSAT program, the challenges of an almost complete turnover of personnel every year, and the dynamics of managing the design, construction, and flying of a satellite every two to three years by a completely student team. Since this program is conducted in the same manner as a typical science and engineering program, students from other academic departments also participate in the program. The program has been augmented by the participation of students from six different academic departments. The addition of this multidisciplinary real-world atmosphere adds an extra dimension of realism to the program. This paper discusses the various solutions the Academy has devised to address the many challenges of conducting a successful program in a highly constrained undergraduate environment.

"Proceedings of the 2004 American Society for Engineering

Education Annual Conference \& Exposition

Copyright C2004, American Society for Engineering Education"
\end{abstract}




\section{Introduction}

The FalconSAT program at the United States Air Force Academy (USAFA) gives undergraduate students a chance to "Learn Space by Doing Space" through a capstone course in the Astronautics Department. This program allows cadets to gain real-world experience with satellite system design, assembly, integration, testing, and operations within the context of a two-semester engineering course. Another goal of the program is to provide a useful platform for Air Force and Department of Defense (DoD) space experiments. Through FalconSAT participation, cadets are given a hands-on opportunity to apply the tools developed in a classroom to a real program, ideally preparing them for the situations they may encounter as officers and as engineers after graduation. Just as any space mission is multi-disciplinary, select students from the Management, Mechanical Engineering, Electrical Engineering, Computer Science, and Physics Departments; participate with the Astronautical Engineering majors in the program. This program uses an evolutionary design approach in which cadets employ or refine cuttingedge technologies and procedures developed by their predecessors. Lessons learned are then captured and help USAFA build a catalog of technical procedures for future missions. Because there is almost a $100 \%$ turnover every year, documentation is crucial to the success of the program[1].

After a brief history of the program, this paper will discuss the approach used to conduct a successful program, using only mentored undergraduate students to design, develop and operate small satellites of nano-satellite and micro-satellite size. All of these satellites have Air Force and DoD space experiments as their mission.

\section{Background}

The USAF Academy started experimenting with small satellites via cadet-built prototypes that were "launched" on high altitude balloons. These projects gave the students immediate, hands-on experience and allowed the Astronautics Department to gradually evolve the curriculum to accommodate increasingly more ambitious space projects. This initial development culminated in the launch of FalconGold in October 1997. FalconGold was a $15 \mathrm{Kg}$ fixed, secondary payload on an Atlas-Centaur launch vehicle. The mission of FalconGold was to determine whether GPS signals could be detected above the GPS constellation. FalconGold relayed GPS data for 15 days prior to battery depletion. Successful operations and data recovery from FalconGold concluded that GPS signals could be used for orbit determination, even beyond the altitude of the GPS constellation[2]. With the space launch of FalconGold, the groundwork was in place to continue the program exclusively with space launched satellites[3].

The Academy's first "free-flyer" satellite, FalconSAT-1 (FS-1), was a $52 \mathrm{Kg}$ satellite launched on January 14, 2000 aboard the first Minotaur launch vehicle (a modified Minuteman II ICBM) along with several other university-built micro-satellites. FS-1 flew the DoD-supported Charging Hazards and Wake Studies-Long Duration (CHAWS-LD) experiment which was designed to measure electric potential created by a

"Proceedings of the 2004 American Society for Engineering Education Annual Conference \& Exposition

Copyright C2004, American Society for Engineering Education" 
spacecraft's wake to examine how charging varies throughout an orbit. The CHAWS-LD sensor was designed to assess the hazards for spacecraft operations in the wake of larger bodies. Unfortunately, a power system problem became apparent soon after deployment. Despite repeated attempts to recover the spacecraft by the cadet-faculty operations team, the mission was declared a loss after only one month [3].

Although it was considered a technical failure, FS-1 represented an academic success for the program because cadets participated from "cradle to grave" in a real-world mission with an all too real-world outcome. Cadets designed and built FS-1's payload and subsystems, which were integral in the mission operations from devising operations plans to participating in the launch campaign. Cadets also manned the Academy's ground station during overhead passes of a satellite not operating under normal conditions. Cadets involved with trouble-shooting the anomalies soon after deployment certainly gained deep insight into system functions and operations[3].

\section{A More Standardized Program}

The lessons learned from FS-1 motivated a significant structural change to the program, with the intention of building a program first and a satellite second. Thus, the new approach was to focus on building up infrastructure, including design and development tools that can serve as a firm foundation to allow the design to evolve steadily over the course of several missions. The FS-2 design effort was aimed at developing a flexible platform that can be readily adapted and enhanced to meet future payload requirements and secondary launch opportunities[1].

Part of this new approach was a major effort to bound the problem faced by the students. To do this, the program has leveraged research at the Surrey Space Center at the University of Surrey, UK. In June 2000, engineers there launched the first SNAPSat satellite, a $6.5 \mathrm{~kg}$, highly functional, spacecraft with 5 imaging cameras, 3-axis attitude control and a propulsion system [4]. SNAPSat represents the culmination of research into open spacecraft architectures. By buying into this SNAPSat architecture, the Academy program has achieved an "out-of-the-box" solution for several critical subsystems; including power, communications and, most important, data handling [3].

Using one set of SNAP hardware, the FS-1 Avionics Simulation Testbed (FAST) was established in Fall 2000. FAST provides both a long-term facility for cadets to gain hands-on experience with spacecraft hardware and software, as well as overall program risk reduction by providing a facility for subsystem, software, and operational procedures development and testing. While the use of commercial off the shelf (COTS) hardware such as SNAP has eased the design problem in many respects, considerable effort still remains in the areas of payload design and development, structures, attitude control, thermal control, solar panels, testing and operations-more than enough to challenge even the most ambitious undergraduate students[3].

A concerted effort was also made to involve cadets from a variety of departments, not just Astronautics, to expand the knowledge base of participants and give every cadet,

"Proceedings of the 2004 American Society for Engineering

Education Annual Conference \& Exposition

Copyright @2004, American Society for Engineering Education" 
regardless of their major, an opportunity to contribute to the program. This approach better reflects how technical programs in the Air Force are conducted, involving engineers, scientists, managers, technical writers and other experts from a variety of fields. Teaming scientists with a keen interest in designing space experiments with engineers who want to build missions, provided great synergy to the program. The CHAWS-LD experiment flown on FS-1 was conceived and built by faculty and students from the USAF Academy's Physics Department. Since then, select computer science, electrical engineering, mechanical engineering, and management majors have joined the program. This change has not only given an interesting scientific focus to the missions, but has brought real-world credibility. The experiments on all FalconSAT missions compete for recognition across DoD for approval by the DoD Space Experiments Review Board (SERB). Such credibility gives the added bonus of critical additional funding, and all-important space launch opportunities [3].

With this real-world focus, real-world funding and real-world visibility, it has become ever more important to run the program using real-world tools. Chief among these are rigorous systems engineering processes including technical reviews. The DoD mandates a tailored acquisition sequence for all its programs that closely follows the IEEE Standard for Application and Management of the Systems Engineering Process [2]. This process begins with requirements analysis and culminates in system deployment. Along the way, major milestones in the form of formal technical reviews are conducted. In addition to multiple status reviews, these include:

- Preliminary Design Review (PDR)

- Critical Design Review (CDR)

- Final Readiness Review (FRR)

It should be emphasized that the cadets do all of the briefing in the many informal and semi-formal status reviews conducted continually throughout the program. The Preliminary Design Review (PDR) is a formal briefing with the objective of gaining permission to proceed with the fabrication and test of subsystems. The cadets are not allowed to acquire materials or begin construction until all action items are closed from the PDR. This review forces the cadets to have a detailed and well thought out design before committing funds and effort to fabrication and testing of subsystems. The introduction of the importance of cost is a shocker to the student who is used to always using the textbook solution. This practicality is a very maturing process for many young engineers and introduces them to the importance of maintaining good relationships with the management side of the program. It also introduces them to the complexity of a program and the tremendous responsibilities and pressures on a program manager. They thus come to understand the problem of how to produce a successful program under budget [3].

The Critical Design Review (CDR) is a formal, multi-day briefing to reviewing experts from outside of the Air Force Academy. The objective of the CDR is to gain permission to proceed with the integration of all subsystems and the performance of operational/field testing of the total system. As usual the cadets are the briefers - which amounts to an

"Proceedings of the 2004 American Society for Engineering Education Annual Conference \& Exposition Copyright @2004, American Society for Engineering Education" 
oral examination of their project. Just as you really learn a subject when you are required to teach it, being subjected to questions throughout the student's briefing by outside experts, stimulates increased understanding of the subject -- hopefully before the briefing, but always afterwards3].

The Final Readiness Review (FRR) is the equivalent of the Prototype Acceptance Demonstration (PAD) in the DoD procurement program. The FRR is a formal review that all of the requirements of the program have been fulfilled. This includes the thermal bake-out testing, the shake test, etc. The satisfactory completion of this review means the satellite is ready to be launched[3].

By its nature, any design class is open ended and difficult to program lesson-by-lesson as a traditional lecture-based course would be. However, by requiring students to follow prescribed, industry-standard systems-engineering processes, some formal structure can be imposed on the semester and the design reviews serve as major deliverables for grading purposes [3].

Another improvement to the program was that in addition to using COS items as a foundation for the design of the satellite, the cadets were required to build three models of the satellite during the satellite development for a single mission.

1. An Engineering Model (EM) to make sure all of the components fit and are compatible for the mission.

2. A Qualification Model (QM) which has all of the characteristics of the Flight Model and is tested to above the limits of all aspects required of the Flight Model.

3. A Flight Model (FM) which is the satellite that will be flown in space.

Each model of the satellite must complete the entire review process through FRR, before starting the fabrication and testing of the next model of the satellite. This procedure is a vast improvement in assuring the reliability of the satellite [5].

\section{FalconSAT-2 (FS-2)}

The improved program was used in the design and development of FalconSAT-2 (FS-2), a nano-satellite with a mass of $19.5 \mathrm{Kg}$ and a volume of approximately $30,000 \mathrm{~cm}^{\wedge} 3$. The mission of FS-2 is using the Micro-Electro Static Analyzer (MESA) to detect and characterize plasma bubbles in the ionosphere which can interfere with Global Positioning System (GPS) signals and other military-critical communications. A photograph of the MESA sensor is shown in Figure 1. 


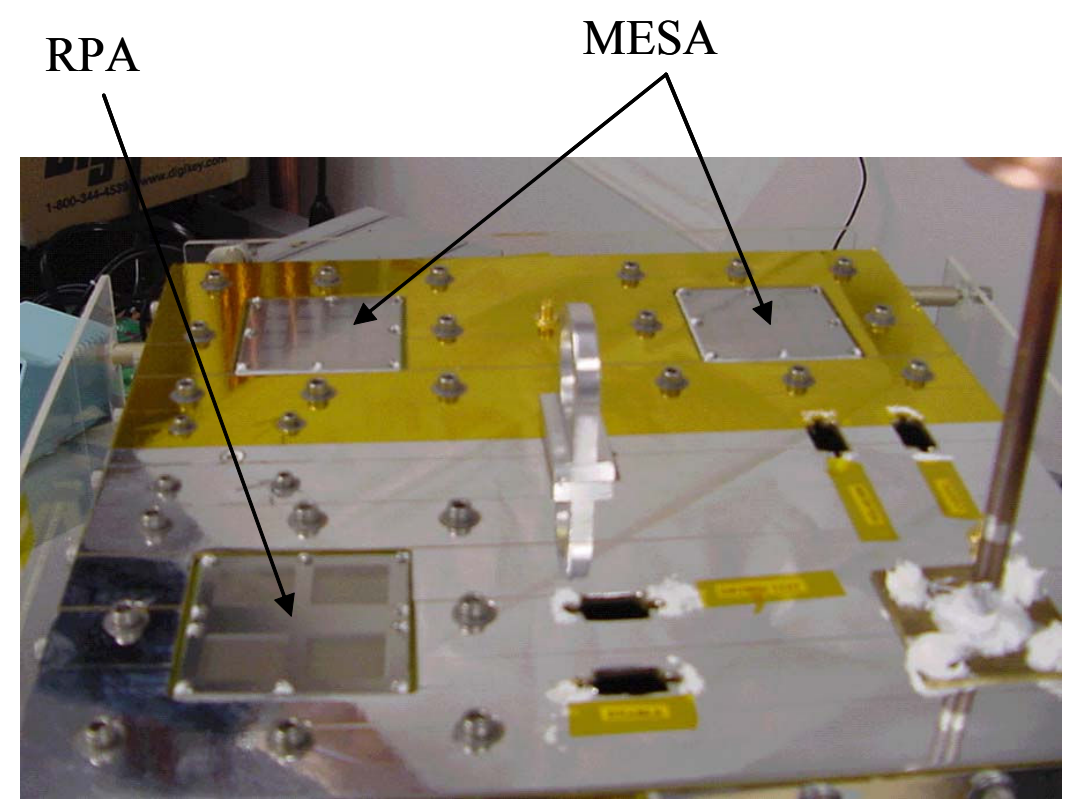

Figure 1: Arrangement of the MESA patch sensors on the top panel of FS-2.

FS-2 was scheduled to be launched on the NASA Space Shuttle flight STS-114. The next shuttle mission scheduled after the Columbia mission. FS-2 is space flight ready and will be launched as soon as the NASA shuttle missions are resumed. Figures 2 and 3 illustrate the completed FS-2 satellite. Figure 4 shows cadets performing thermal vacuum testing in a clean room environment.

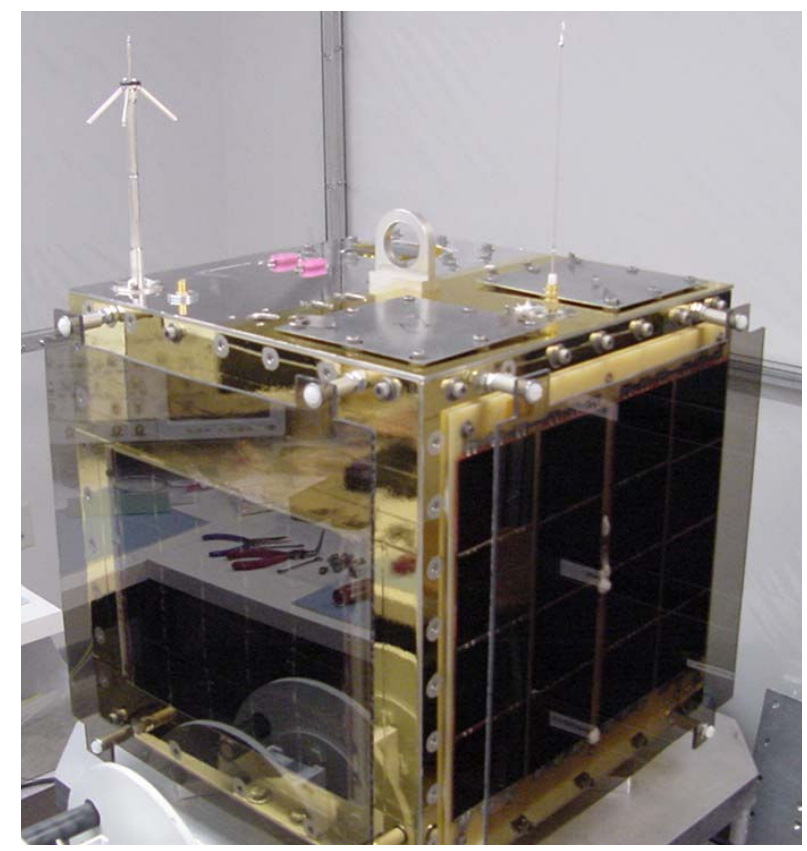

Figure 2: FS-2 External View

"Proceedings of the 2004 American Society for Engineering Education Annual Conference \& Exposition 


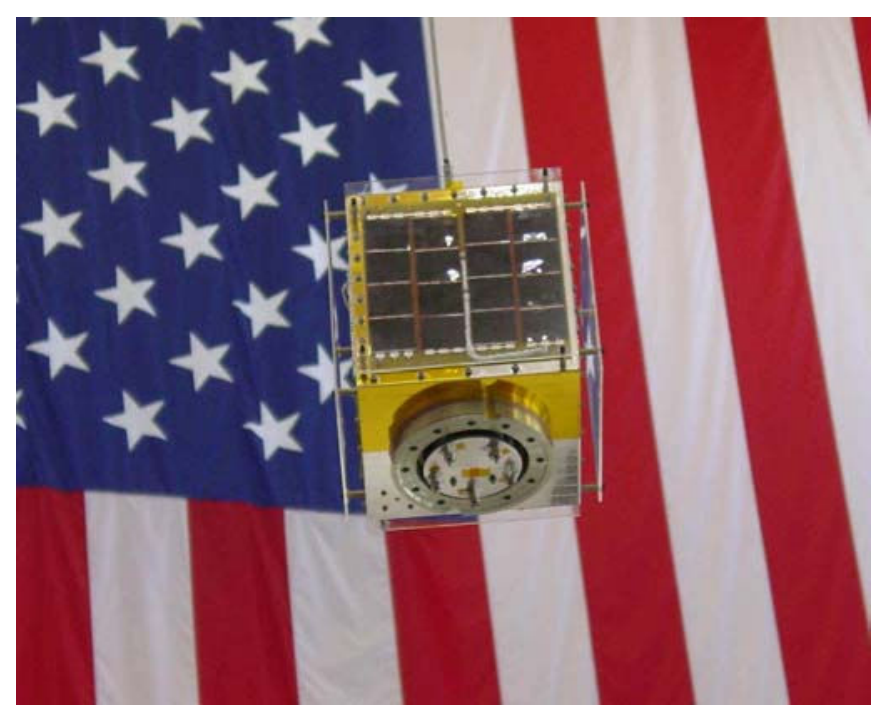

Figure 3. FS-2 Ready for Flight

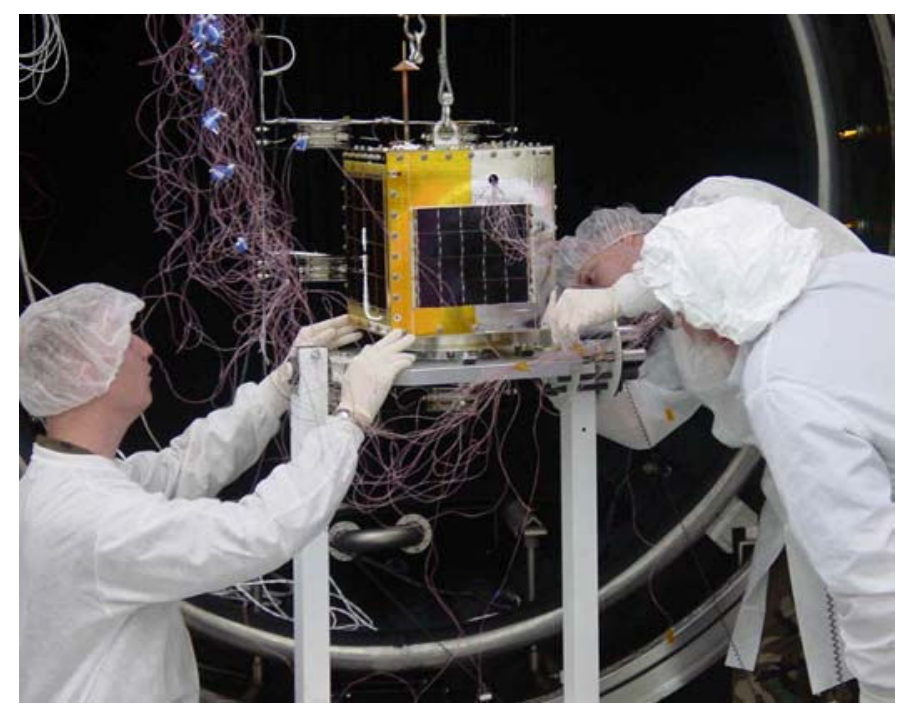

Figure 4. Cadets Performing FS-2 Thermal Vac Testing

The specific scientific objective of the FS-2 mission is to use a Miniature Electrostatic Analyzer (MESA) to:

1. Investigate the morphology of plasma depletions in the F region ionosphere.

2. Demonstrate the utility of MESA in the measurement of thermal ionospheric electrons.

3. Investigate the structure and evolution of ionospheric plasma bubbles. 


\section{FalconSAT-3 (FS-3)}

FS-3 is a nano-satellite with a mass of about $50 \mathrm{Kg}$. FS-3 has completed the Engineering Model testing. Construction of the Flight Model will start in the Fall Semester of 2004. FS-3 is scheduled to launch in 2006. Figure 5 is a picture of the CAD Model of FS-3 [6].

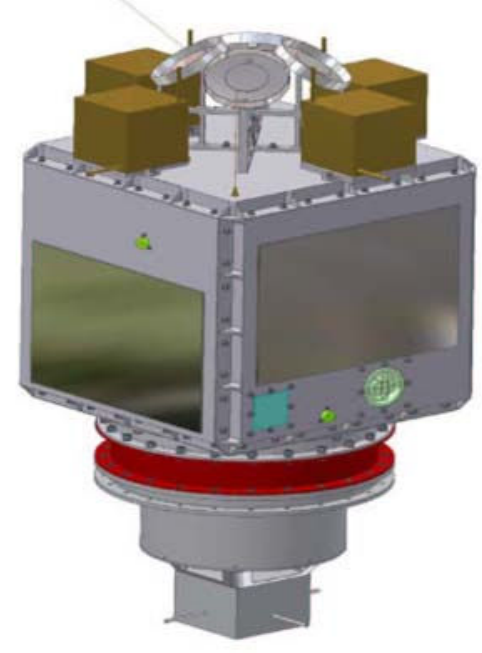

Figure 5. A CAD Model of FalconSAT-3 (FS-3).

The three DoD Space Evaluation Review Board (SERB) approved scientific objectives of the FS-3 mission are:

1. Micro Propulsion Attitude Control System (MPACS) evaluation - An advanced electric propulsion technology powered by the spacecraft's Electrical Power System. The system is designed to provide affordable, effective attitude control torque in order to supplement a typical small satellite's Attitude Determination and Control Subsystem (ADCS).

2. Using a Flat Plasma Spectrometer (FLAPS) to characterize the formation, propagation, and decay of ionospheric plasma bubbles more accurately than MESA on FS-2.

3. Characterization of the Plasma Local Anomalous Noise Environment (PLANE) surrounding a space vehicle. The PLANE experiment will investigate the localized plasma environment caused by the spacecraft's movement through the ionosphere. More specifically, this experiment will compare the spacecraft's motion and associated charging with the ambient plasma charge as the satellite's experiment face travels through ram-side plasma. 
FS-3 will also carry two additional experiments.

4. A Shock Ring vibration isolation system to reduce launch vehicle shock on payloads.

5. Gravity Gradient Boom which uses Elastic Memory Composite (EMC) Hinge technology for proof of concept for deployment of various appendages (e.g., solar arrays, antenna arms, and more complex gravity gradient booms)[5].

Table 1 summarizes the various milestones in the FalconSAT Program thus far.

Table 1. Summary of FalconSAT Program Milestones.[3]

\begin{tabular}{||l|l|l|l||}
\hline \hline DATE & EVENT & SATELLITE & MISSION \\
\hline May 1995 & Balloon Flight & USAFASAT-B & Attitude Control Demonstrator \\
\hline Mar 1996 & Balloon Flight & Glacier & GPS \& Magnetometer Experiment \\
\hline Sep 1996 & Balloon Flight & PHOENIX & Laser Communication Demo \\
\hline Apr 1997 & Balloon Flight & FalconGold & GPS Signal Capture \\
\hline Oct 1997 & Space Launch & FalconGold & GPS Signal Capture \\
\hline Jan 2000 & Space Launch & FalconSAT-1 & CHAWS-LD \\
\hline Present & $\begin{array}{l}\text { Ready for } \\
\text { Space Launch }\end{array}$ & FalconSAT-2 & MESA \\
\hline Present & $\begin{array}{l}\text { Construction \& } \\
\text { Testing of } \\
\text { Qualification } \\
\text { Model }\end{array}$ & FalconSAT-3 & $\begin{array}{l}\text { MPACS } \\
\text { FLAPS } \\
\text { PLANE }\end{array}$ \\
\hline
\end{tabular}

\section{Satellite Operations}

Obviously when satellites are launched, there must be a ground station for the operation of the satellite while in flight. While planning the launch of FS-1, the Department of Defense Space Test Program needed a ground station in the United States for a microsatellite named PICOSAT. PICOSAT was built by Surrey Satellite Technology Ltd (SSTL) in Guildford, UK and was partially funded through the DoD Foreign Comparative Testing Program. This ground station was located in the USAFA Space Systems Research Center (SSRC). The ground station for FS-1 was collocated with the PICOSAT Ground Station and has developed into the present system. A floor plan of the present FalconSAT Ground Station is illustrated in Figure 6. The PICOSAT section of the Ground Station is an automated system and usually does not require manning.

The line-of-sight antennas are located on the roof of the building. Therefore, the communication with the satellite is limited to the time it is in view of the antennas. This contact time varies from approximately one to ten minutes per pass. The satellite's ground track is such that there are times that there are multiple orbits where the satellite is not in view of the Ground Station at all. This can result in several hours between passes. A very detailed description the FalconSAT Ground Station, its operation and the training program for the cadets who man it is presented in Spacemanship at the United States Air

\footnotetext{
"Proceedings of the 2004 American Society for Engineering

Education Annual Conference \& Exposition

Copyright @2004, American Society for Engineering Education"
} 


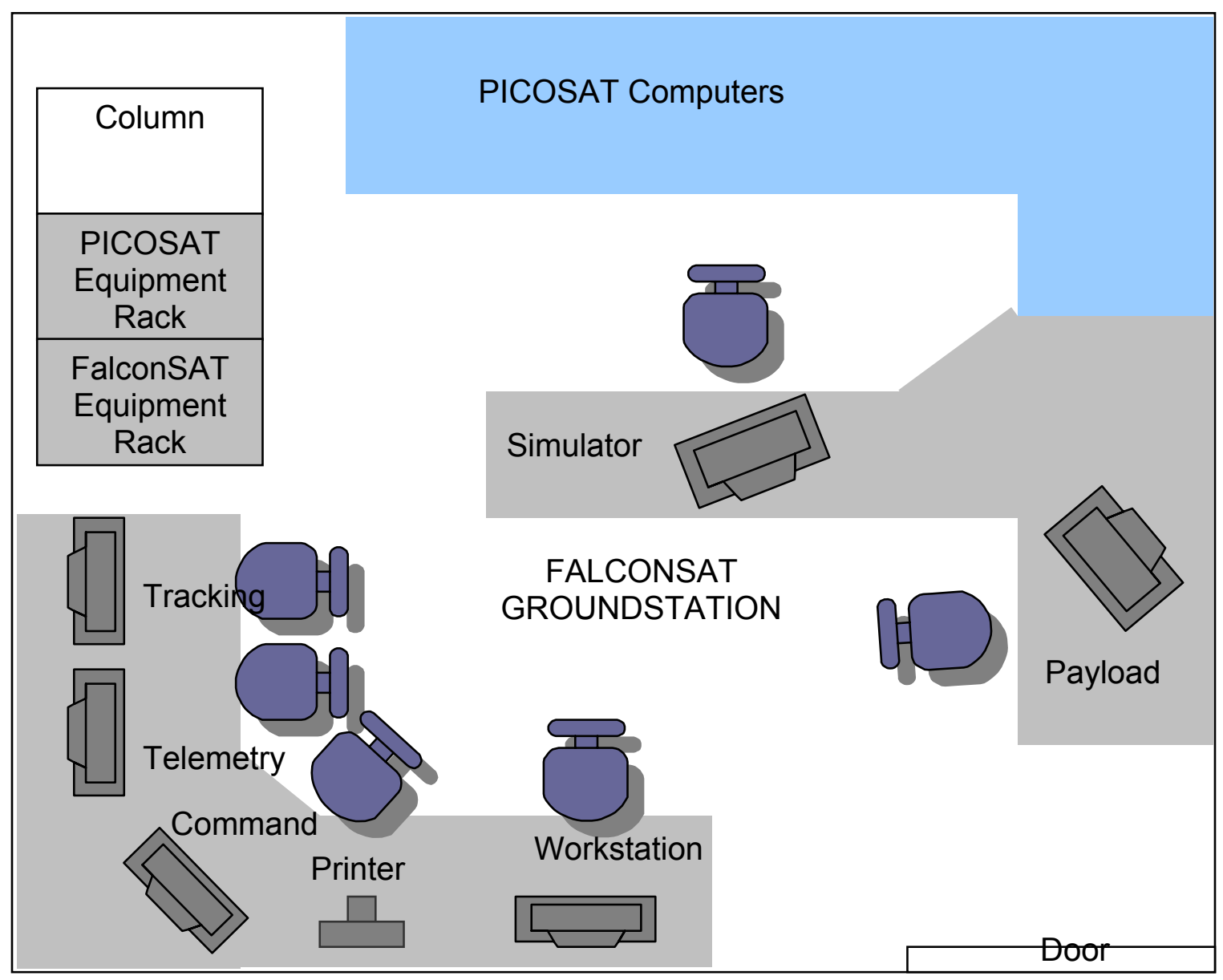

Figure 6. USAFA FalconSAT Ground Station Floor Plan

Force Academy: Developing a Satellite Ground Station Crew Training Program for Non-Technical Students, by Swanson, et.al.[7]

\section{Course Grading and Cadet Reaction to the Program}

Assigning individual grades to a multidisciplinary group project of this size is a challenge. The grading system developed includes the input of peer evaluations by the members of each subdivision team and evaluations by the faculty mentors of these teams. The final grades are assigned by the senior faculty members in charge of the course.

The overall reaction of the cadets to the program has been very positive even though everyone, including the faculty, is a volunteer. Many cadets come into their own in this type of course. Comments like "Loose structure of the class was great, allowed individual initiative" and "Major Miller is an excellent mentor. He cares about his student's success. I have enjoyed the class \& the instructors" are examples of optional

"Proceedings of the 2004 American Society for Engineering Education Annual Conference \& Exposition 
written comments on the student critiques. The student response was high in all 21 categories. In regards to the answers to the question, "Intellectual challenge and encouragement of independent thought were?" the course scored in the top $2 \%$ of all the courses taught at the Air Force Academy. The course also scored in the top $2 \%$ of all the courses taught in the Engineering Division for; 1.) "Relevance and usefulness of course content" and 2.) Encouragement given students to express themselves and participate. The amount learned in the course and the instructor's effectiveness in facilitating the student's learning in the course were in the top 5\%. When you consider that these cadets are working very hard right up to graduation of their senior year while other classmates are having an easy semester, these comments are very encouraging as to the quality of Air Force officers and engineers that these cadets will become [8].

\section{Customer Reaction to the Program}

One of the satisfying aspects of this program is the fact that the mission is a real space mission and not just a textbook exercise. The program is continually reviewed by outside experts and evaluated against the work of the real space community, not just academia. The comments by outside space experts and some of the customers of the program who were the reviewing officials at a recent Critical Design Review (CDR) of FalconSAT-3 were very positive: "Very comprehensive in terms of SV subsystem/elements covered." "Team was well prepared, organized, and used the right level of complexity to address technical issues." "Cadets were very professional." "The cadets did 95\% of the briefing." In response to the question, "On a scale of 1 to 10,1 being the worst design review you've ever seen and 10 being the best, how would you rate this CDR?", there were responses of 7,8 and 9 and one comment of "10 if based on university satellite programs and an 8.5 compared to all satellite programs".

\section{Conclusions}

Of course, all programs are judged on their results. The physical result of the FalconSAT program is of world-class quality. The cadets do what most nations cannot do. The real product of the FalconSAT program is the professional Air Force officers who have had the "Learning Space by Doing Space" experience while at the Air Force Academy. The exposure to solving ill defined problems in the FalconSAT program prepares the cadet for the challenges of a professional military career. The space aspect of the program prepares them to join the cadre of space professionals who have enabled the United States Air Force to dominate the globe in terms of air and space power.

\section{Acknowledgements}

The authors would like to acknowledge the contributions to the success of this program by all of the cadets and mentors who have been involved in the program. In particular, the dedicated hard work of Mr. Jim White, Mr. John Clark, Master Sergeant Phil Maes, Technical Sergeant Chad Bruce, and Technical Sergeant Benjamin Hazen is greatly appreciated. If you enjoyed reading this paper, credit our editors, Dr. Fred Kiley and Ms. Lynnette Kaffine.

\footnotetext{
"Proceedings of the 2004 American Society for Engineering Education Annual Conference \& Exposition Copyright C2004, American Society for Engineering Education"
} 


\section{Bibliography}

1. Sellers, Jerry J. and Timothy J. Lawrence, "Building a Cadre of Space Professionals: Hands-on Space Experience at the USAF Academy," $1^{\text {st }}$ Responsive Space Conference, Redondo Beach, CA, 1-3 April 2003.

2 "FalconGold Program Mission Overview," this information is available on the web at www.usafa.af.mil/dfas/research/falcongold/falcongold.html.

3. Sellers, Jerry J., and Doug Bayley, "Spacecraft Design, Development and Operations in Just Five Hours per Week: Experience from the USAF Academy FalconSAT Program," Proceedings of the 2001 American Society of Engineering Educators Annual Conference \& Exposition, 2001.

4. Stuart Stanton and Jerry Sellers, "Modeling and Simulation Tools for Rapid Space System Analysis and Design: FalconSat-2 Applications," presented at the 2001 IEEE Aerospace Conference, Big Sky, MT, March 2001.

5. Richie, David J., Jerry J. Sellers, "FalconSAT-3: A Military University Satellite for Innovative Department of Defense Experiments," 2003 Core Technologies for Space Systems Conference, Colorado Springs, CO, 4-6 November, 2003

6. FalconSAT-3 Critical Design Review Slide Package. 30 September - 01 October 2003.

7. Swanson, David, Kenneth E. Siegenthaler, David J. Barnhart, Jerry J. Sellers, David J. Richie, and Else Bruno, "Spacemanship at the United States Air Force Academy: Developing a Satellite Ground Station Crew Training Program for Non-Technical Students," ," Proceedings of the 2004 American Society for Engineering Education Annual Conference \& Exposition," Salt Lake City, UT, June 2004.

8. United States Air Force Academy Student Critiques for Astro 436/437, 2002-2003.

\section{Biographies}

KENNETH E. SIEGENTHALER is an Associate Professor of Astronautics at the U.S. Air Force Academy. Dr. Siegenthaler has a B.S in the Arts \& Sciences from the U.S. Military Academy, a B.S. in Physics from the University of Utah, and a M.S. and a Ph.D. in Engineering Physics from the Air Force Institute of Technology. He is a registered Professional Engineer in the state of Colorado.

JERRY J. SELLERS is an active-duty Lieutenant Colonel in the U.S. Air Force and an Associate Professor of Astronautics at the U.S. Air Force Academy. He has a B.S. from the U.S. Air Force Academy, M.S. from U. of Houston, M.S. from Stanford University and a Ph.D. from the University of Surrey, UK. Currently he is Director of the USAF Academy Space Systems Research Center in Colorado Springs, CO.

DAVID J. RICHIE is an active-duty Captain in the U.S. Air Force and an Assistant Professor of Astronautics at the U.S. Air Force Academy. He has a B.S. in Astronautics from the U.S. Air Force Academy and an M.S. in Astronautical Engineering from the Georgia Institute of Technology. He is presently Laboratory Director for the Department of Astronautics at the U.S. Air Force Academy.

TIMOTHY J. LAWRENCE is an active-duty Major in the U.S. Air Force and an Assistant Professor at the U.S. Air Force Academy. He has a B.S from the U.S. Air Force Academy, an M.S. from MIT, and a Ph.D. from the University of Surrey, UK. Currently he is the Navigation, Guidance and Control Division Chief of the Department of Astronautics at the United States Air Force Academy.

"Proceedings of the 2004 American Society for Engineering

Education Annual Conference \& Exposition

Copyright @2004, American Society for Engineering Education" 